\title{
Rastreo satelital de las hembras de tortuga blanca Chelonia mydas y evaluación de sus ámbitos hogareños en la costa norte de la península de Yucatán, México
}

Satellite tracking of green turtle females Chelonia mydas and the evaluation of their home ranges in the north coast of the Yucatán Peninsula, Mexico

\section{Dennis Méndez', Eduardo Cuevas², Jorge Navarro', Blanca I. González-Garza ${ }^{3}$ y Vicente Guzmán-Hernández ${ }^{4}$}

\author{
${ }^{1}$ Universidad Autónoma de Yucatán, Campus de Ciencias Biológicas y Agropecuarias, Carretera Mérida-Xmatkuil km. 15,5, \\ A.P. 4-116 Itzimná, Yucatán, México \\ 2Pronatura Península de Yucatán, A.C., Calle 32 No. 269 x 47 y 47 A Colonia Pinzón II. C.P. 97207, Mérida, Yucatán, \\ México.ecuevas@ pronatura-ppy.org.mx \\ ${ }^{3}$ Centro de Investigación y de Estudios Avanzados, Instituto Politécnico Nacional, Unidad Mérida, Km. 6 Antigua Carretera \\ a Progreso, C.P. 97310, Mérida, Yucatán, México \\ ${ }^{4}$ Área de Protección de Flora y Fauna Laguna de Términos, Comisión Nacional de Áreas Naturales Protegidas, Av. Adolfo \\ López Mateos, Prolongación Playa Norte, C. P. 24140, Ciudad del Carmen, Campeche, México
}

\begin{abstract}
From the 32 worldwide post nesting Chelonia mydas sub populations 2 are found in the Mexican Coasts, one of them in Michoacan (Pacific Ocean) and the other in the Yucatán Peninsula (Atlantic Ocean). Lack of information about migration patterns, home ranges and anthropogenic interaction are factors that contribute to the intrinsic vulnerability of $\mathrm{C}$. mydas populations anywhere around the world. The objective of this investigation was to identify migration routes and critical marine habitats for post nesting C. mydas individuals in the Yucatán Peninsula. Between June 2011 and January 20124 C. mydas post nesting individuals were tracked using satellite telemetry, 2 of them from continental beaches and the other 2 from insular ones. A geographic information system was used to integrate the collected information and used to identify migration routes for $\mathrm{C}$. mydas as well as to estimate their home ranges. Three turtles moved and remained over the marine platform in the Banco de Campeche in Mexican territory, while one of the turtles migrated outside Mexican waters to Dry Tortuga (Florida, USA). The home ranges areas used by nesting females in continental beaches were significantly larger and further from the coast compared to those registered for females tracked from insular beaches. Based on the results we recommend management strategies planning for conservation of $C$. mydas in the Yucatán Peninsula.
\end{abstract}

Key words: Critical habitats, continental and insular home ranges, migratory patterns

Resumen.- A nivel mundial de las 32 subpoblaciones de Chelonia mydas post-anidadoras, 2 se encuentran en las costas de México, una en Michoacán (Océano Pacífico) y la otra en la península de Yucatán (Océano Atlántico). La carencia de información sobre patrones migratorios, ámbitos de hogar, e interacción antropogénica, son factores que contribuyen a la vulnerabilidad intrínseca de las poblaciones de C. mydas en cualquier parte del mundo. El objetivo de esta investigación fue identificar las rutas migratorias y hábitats críticos marinos para individuos de C. mydas post-anidadoras en la península de Yucatán. Entre junio de 2011 y enero de 2012, 4 individuos de C. mydas post-anidadoras se rastrearon utilizando telemetría satelital, 2 desde playas continentales y 2 desde playas insulares. Se utilizó un sistema de información geográfica para integrar la información recabada, y utilizándola para identificar las rutas migratorias de C. mydas, así como estimar sus áreas de ámbitos de hogar. Tres tortugas se desplazaron y se mantuvieron sobre la plataforma marina del Banco de Campeche en territorio Mexicano y una migró fuera de aguas nacionales Mexicanas hasta llegar a Dry Tortuga (Florida, USA). Las áreas abarcadas de los ámbitos de hogar por hembras anidadoras en playas continentales fueron significativamente mayores y se ubicaron a mayor distancia de la costa respecto a los registrados por las hembras rastreadas desde playas insulares. Con base en los resultados se recomienda la planificación de estrategias de manejo para la conservación de C. mydas en la península de Yucatán.

Palabras clave: Hábitats críticos, ámbitos de hogar continental e insular, rutas migratorias 


\section{INTRODUCCIÓN}

En la costa del Atlántico mexicano, la tortuga marina de la especie Chelonia mydas (Linnaeus, 1758) es conocida como tortuga blanca (Pritchard \& Mortimer 1999, Seminoff 2004). En el Gran Caribe, la región de la península de Yucatán alberga algunas de las subpoblaciones más importantes de esta especie de las 32 reportadas a nivel mundial (Groombridge 1982, Groombridge \& Luxmoore 1989). Al igual que las otras 6 especies de tortugas marinas, C. mydas tiene un ciclo de vida complejo, siendo de vital importancia para su desarrollo hasta la etapa adulta, el uso de varios hábitats marinos, entre ellos, playas, áreas marinas costeras neríticas, arrecifes de coral y aguas oceánicas (Groombridge \& Luxmoore 1989, Eckert \& Abreu-Grobois 2001).

Durante el siglo pasado, en el Golfo de México y la Región del Caribe, ha existido la explotación comercial de productos obtenidos de individuos de C. mydas, que redujo drásticamente colonias de subpoblaciones en su área de distribución natural, evidenciándose bajos índices de diversidad genética entre colonias de algunas subpoblaciones (McClenachan et al. 2006). Se han realizado estudios moleculares en hembras postanidadoras de tortuga blanca para identificar y evaluar diferencias entre colonias de una misma subpoblación y de esta a otras subpoblaciones distribuidas en zonas tropicales (Peare \& Parquer 1996). Un estudio realizado por Seminoff (2004) a nivel mundial para la evaluación del estatus de conservación de esta especie reportó un decremento entre el 48 y $76 \%$ del número de hembras reproductivas en las últimas décadas.

Desde la década de 1960 en aguas territoriales mexicanas del Pacífico, Atlántico, Caribe y del Golfo de México, se conoce sobre capturas dirigidas e incidentales de tortugas marinas por pescadores, debido a la superposición de hábitats críticos marinos de uso común (Lewison \& Crowder 2007). Como medidas de protección, en la década de 1990, el Gobierno de México publicó en el Diario Oficial de la Federación la prohibición de la captura de las 7 especies de tortugas marinas, a la vez que se firmaron otros acuerdos internacionales y nacionales (Márquez 1996, CIT 2012). La norma mexicana NOM-059SEMARNAT-2010 categorizó a $C$. mydas como en peligro crítico de extinción, y CITES desde 1981 la enlistó en el apéndice I, por su parte, la UICN, desde 1982 registró esta especie como en peligro (Groombridge 1982, Seminoff 2004).

En la actualidad, la planificación, conservación y manejo de tortugas marinas, cuenta con herramientas tecnológicas de vanguardia para la identificación de hábitats críticos a través del entendimiento de la ecología espacial. Una de esas herramientas es la telemetría satelital que ha revolucionado el estudio de animales marinos con amplio rango de distribución y que utilizan hábitats poco accesibles para los investigadores.

La relevancia de este tipo de tecnologías es que permite generar información ecológica y biológica básica en diferentes etapas del ciclo de vida de los organismos de estudio. En el caso específico de las tortugas marinas, los rastreos satelitales se han constituido como una herramienta útil en la identificación de los hábitats críticos marinos (Troëng et al. 2005, James et al. 2005) ambientes poco o nada conocidos, donde las tortugas marinas pasan la mayor parte de su vida

Las técnicas tradicionales de marcaje y recaptura con marcas metálicas que tienen el objetivo de evaluar los movimientos de las tortugas en sus áreas marinas (Groombridge \& Luxmoore 1989), fueron complementadas con tecnologías de mayor precisión y costos, como son la telemetría satelital y los sistemas de información geográfica (SIG) (Hoog et al. 2000, Coyne \& Godley 2005), con las cuales es posible identificar rutas migratorias y delimitar ámbitos de hogar de tortugas marinas hembras adultas o individuos juveniles (Fancy et al. 1988).

Los hábitats críticos para C. mydas y otras especies de tortugas marinas, son de vital importancia para completar su ciclo de vida (Bowen et al.1992, Lohmann et al. 2007). Comúnmente, éstas áreas son compartidas entre naciones diferentes (Witt et al. 2011, Hart et al. 2012), por lo que se considera fundamental priorizar acciones de conservación e investigación a escala regional y sin fronteras territoriales, dada la vulnerabilidad de la especie y la existencia de múltiples amenazas a diferentes escalas temporales y espaciales, particularmente por la degradación de hábitats críticos y pesca incidental (Witt et al. 2011, CIT 2012, Hart et al. 2012).

Son pocos los estudios de conservación e investigación realizados para $C$. mydas en la península de Yucatán. Los existentes se han orientado en su mayoría a los hábitats terrestres de playas, dejando vacios de información sobre los hábitats críticos en las áreas marinas costeras (Cuevas et al. 2008). Estudios pioneros de telemetría satelital y análisis espaciales de los hábitats críticos de Eretmochelys imbricata y Caretta caretta post-anidadoras de la península de Yucatán, brindaron información de áreas marinas importantes que posiblemente son utilizadas también por $C$. mydas en la 
región (Byles \& Swimmer 1994, Garduño et al. 2000a,b, Cuevas et al. 2007, Cuevas et al. 2008, Hart et al. 2012). Para C. mydas existen 2 estudios previos de rastreo satelital en el Golfo de México (Garduño et al. 1999, Kinzel 2001).

El presente estudio tuvo como objetivo principal, determinar las rutas migratorias de 4 hembras postanidadoras de $C$. mydas y localizar las áreas utilizadas para su periodo de interanidación y alimentación en la península de Yucatán, además de comparar comportamientos migratorios entre hembras anidadoras en playas continentales y en playas insulares.

\section{Materiales Y MÉTODOS}

\section{Área de ESTUdio}

Para el rastreo por medio de telemetría satelital de 4 individuos de $C$. mydas post-anidadoras, se seleccionaron 4 playas importantes de desove en jurisdicciones estatales de Yucatán y Campeche: 2 en el continente peninsular (Ría Lagartos e Isla del Carmen) y 2 en islas (Arrecife Alacranes y Cayo Arcas) (Fig. 1). Entre la importancia de estas playas, además de que 3 de ellas se encuentran dentro de áreas naturales protegidas, se cuenta la magnitud del número de anidaciones que se registran anualmente, con alrededor de 5,000 anidaciones de $C$. mydas realizadas por $\sim 1,000$ individuos adultos de esta especie de tortuga marina (Ortega et al. 2011¹).

\section{Trabajo de Campo}

Durante patrullajes nocturnos, entre junio y agosto de 2011, 4 tortugas blancas post-anidadoras fueron capturadas al azar para la colocación de igual número de Terminales de Transmisión en Plataforma (PTTs por sus siglas en inglés), para su rastreo satelital. Con la finalidad de diferenciar los individuos, durante el proceso de análisis de datos, se utilizaron nombres para identificarlas: Gertrudis (G), Julieta (J), Marina (M) y Carmelita (C).

Los lugares de captura de los individuos de C. mydas fueron en Ría Lagartos (Gertrudis), Alacranes (Julieta), Arcas (Marina) y Laguna de Términos (Carmelita) (Fig. 1). La adhesión de los PTTs (ST-20 modelo A1010 de Telonics, Inc) en los caparazones de cada individuo fue

Figura 1. Área de estudio en la península de Yucatán. Playas de anidación de Chelonia mydas donde se colocaron las Terminales de Transmisión en Plataforma (PTT por sus siglas en inglés). Zonas continentales (Reserva de la Biósfera Ría Lagartos y Área de Protección de Flora y Fauna Laguna de Términos) y zonas insulares (Parque Nacional Arrecife Alacranes y Cayo Arcas) / Study area in the Yucatán Peninsula. Nesting beaches of Chelonia mydas where Platform Transmission Terminals were deployed. Continental zones (Ria Lagartos Biosphere Reserve and Laguna de Términos Flora and Fauna Protection Area) and insular zones (Arrecife Alacranes National Park and Cayo Arcas)

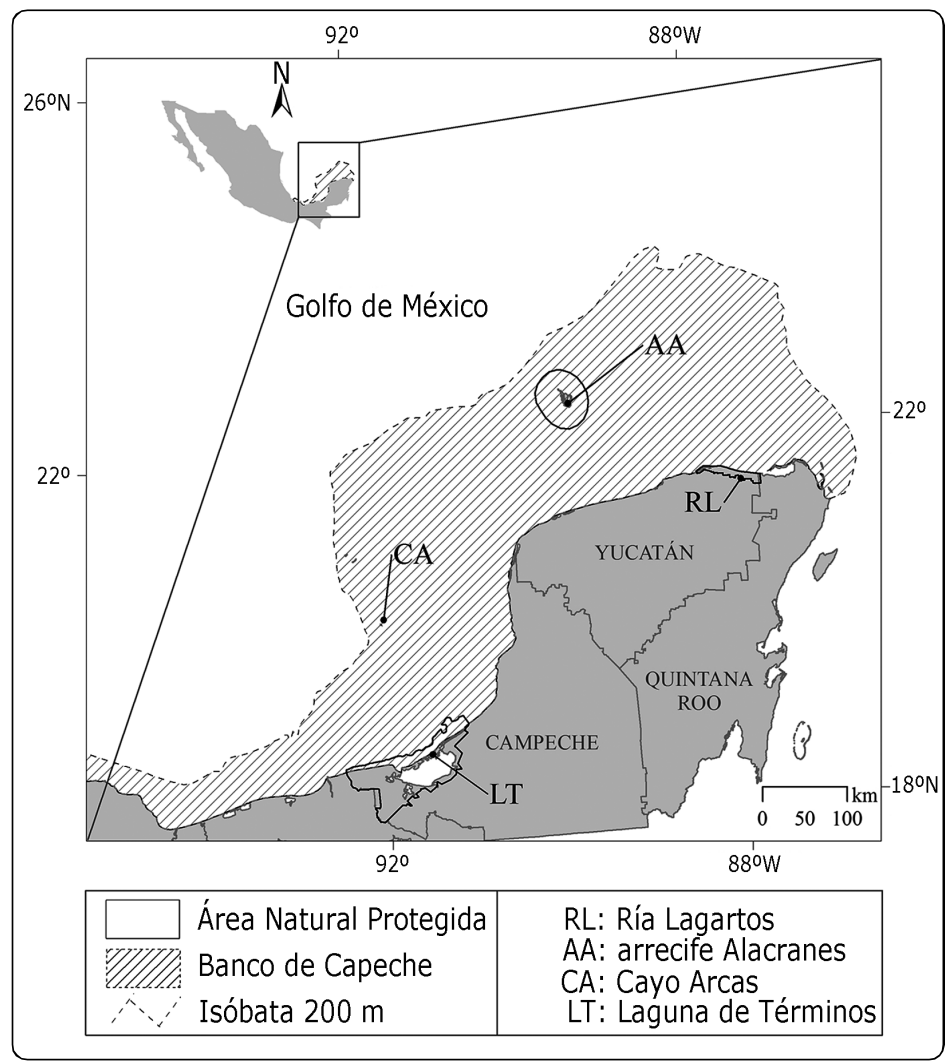

${ }^{1}$ Ortega J, B González \& E Cuevas. 2011. Reporte final temporada 2011. Programa para la conservación de la tortuga marina en la península de Yucatán, 21 pp. Pronatura península de Yucatán A.C., Yucatán. 
realizada con fibra de vidrio y resina de poliéster, a través de metodologías estándar propuestas por Balazs et al. (1996).

El tiempo total de los trabajos de colocación del transmisor por tortuga, fue entre 1,5 y 2,0 h. Durante ese tiempo, se registraron también datos morfométricos y se colocaron marcas metálicas (Inconel modelo 681 en las aletas delanteras), para posteriormente liberar al individuo en el mismo sitio de su captura.

Los rastreos de las hembras variaron en duración: $(\mathrm{G})$ (junio de 2011 a enero de 2012); (J) (julio a noviembre de 2011) (M) (agosto a octubre de 2011) y (C) (agosto a diciembre).

Los PTTs fueron programados para estar encendidos y enviar información durante las $24 \mathrm{~h}$ del día, durante todo el periodo de rastreo. El mecanismo de encendido y apagado de los PTTs fue controlado por un 'interruptor de sal'. El envío de los datos desde los PTTs fue realizada a través del sistema satelital ARGOS, que utilizan el principio doppler para la localización geográfica del transmisor, a través de un conjunto de satélites polares en órbita de la Administración Nacional Atmosférica y Oceánica (NOAA por sus siglas en inglés) del departamento de Comercio de los Estados Unidos de América del Norte.

Para el despliegue y análisis espacial de las coordenadas de las localidades y orientaciones de individuos de $C$. mydas post-anidadoras, se filtraron y seleccionaron las localidades de mayor precisión categorizadas como 3,2,1 y A, las cuales presentan menor margen de error, según el orden mencionado. La precisión respecto a la longitud y latitud de cada localización geográfica según la categoría es de: < $150 \mathrm{~m}$ para 3 , entre 150 y $350 \mathrm{~m}$ para 2 , entre 350 y $1000 \mathrm{~m}$ para 1 . Finalmente, > $1000 \mathrm{~m}$ para 0, A, B y Z (Argos 1996).

Para la ordenación de los datos espaciales y su análisis, se utilizaron los programas ArcView 3.2 (ESRI 1999²) y ArcGIS 10 (ESRI 2010³), con los cuales se integró la información espacial para la elaboración de mapas temáticos de rastreo satelital. Estos procedimientos permitieron una descripción y análisis fundamentado de las rutas de desplazamiento migratorio y áreas de hábitats críticos de $C$. mydas post-anidadoras en la costa norte adyacente a la península de Yucatán.

\section{RASTReo SATElital}

Con la extensión de análisis de movimientos de animales (AMAE por sus siglas en inglés) para ArcView 2.0 (Hooge et al. 2000), se estimaron los ámbitos de hogar de C. mydas post-anidadoras utilizando 2 métodos distintos, (1) El Polígono Mínimo Convexo (PMC), el cual es sensible a datos extremos y tiende a la sobre estimación de áreas de los ámbitos de hogar, pero también es el método más utilizado por su fácil interpretación y por la posibilidad comparativa con investigaciones similares; y (2) el de probabilidades de ocurrencia para datos no paramétricos de Kernel Fijos (KF) (al 50 y 95\%), metodología que determina áreas de ámbitos de hogar con mayor precisión que la anterior (Worton 1989, Yasuda \& Arai 2005).

Las áreas obtenidas por ambos métodos, fueron sometidas a pruebas de distribución normal (Shapiro-Wilk, con un nivel de significación de 5\%) para evaluar la distribución de los datos.

Se realizaron análisis comparativos no paramétricos (Kruskall Wallis y pruebas post-hoc de Dunn, con un nivel de significación de $\alpha=0,05$ ) (Day \& Quinn 1989) para evaluar diferencias entre: áreas de cobertura de ámbitos de hogar delimitados por el PMC y KF (50 y 95\%) cuantificados para tortugas anidadoras en playas continentales y en playas insulares; y sobre la distancia de localidades de $C$. mydas a la costa, diferenciando entre individuos anidadoras en playas continentales e insulares.

Para los análisis espaciales, se ajustaron los datos de las localidades a unidades métricas Transversales de Mercator (UTM) proyectadas en un sistema de coordenadas geográficas mundial de 1984 (WGS84). Dicho ajuste fue realizado para las zonas $15 \mathrm{~N}, 16 \mathrm{~N}$ y 17 $\mathrm{N}$ para las localidades en Campeche, Yucatán y Florida (USA) respectivamente.

\section{NAVEGACIÓN Y RUMBo}

Se realizó un análisis descriptivo de las tendencias en la navegación y orientación de las tortugas blancas durante su fase migratoria con herramientas de estadística circular utilizando el programas Oriana 4 (Kovach 2012) 4

\footnotetext{
${ }^{2}$ ESRI. 1999. Arcview 2.0. Environmental Systems Research Institute, Inc. New York.

${ }^{3}$ ESRI. 2010. Arcmap 10.0. Esri Inc., New York. <http://www.arcgis.com/about>

${ }^{4}$ Kovach CS. 2012. Oriana version 4.01. Kovach Computing Services
} 


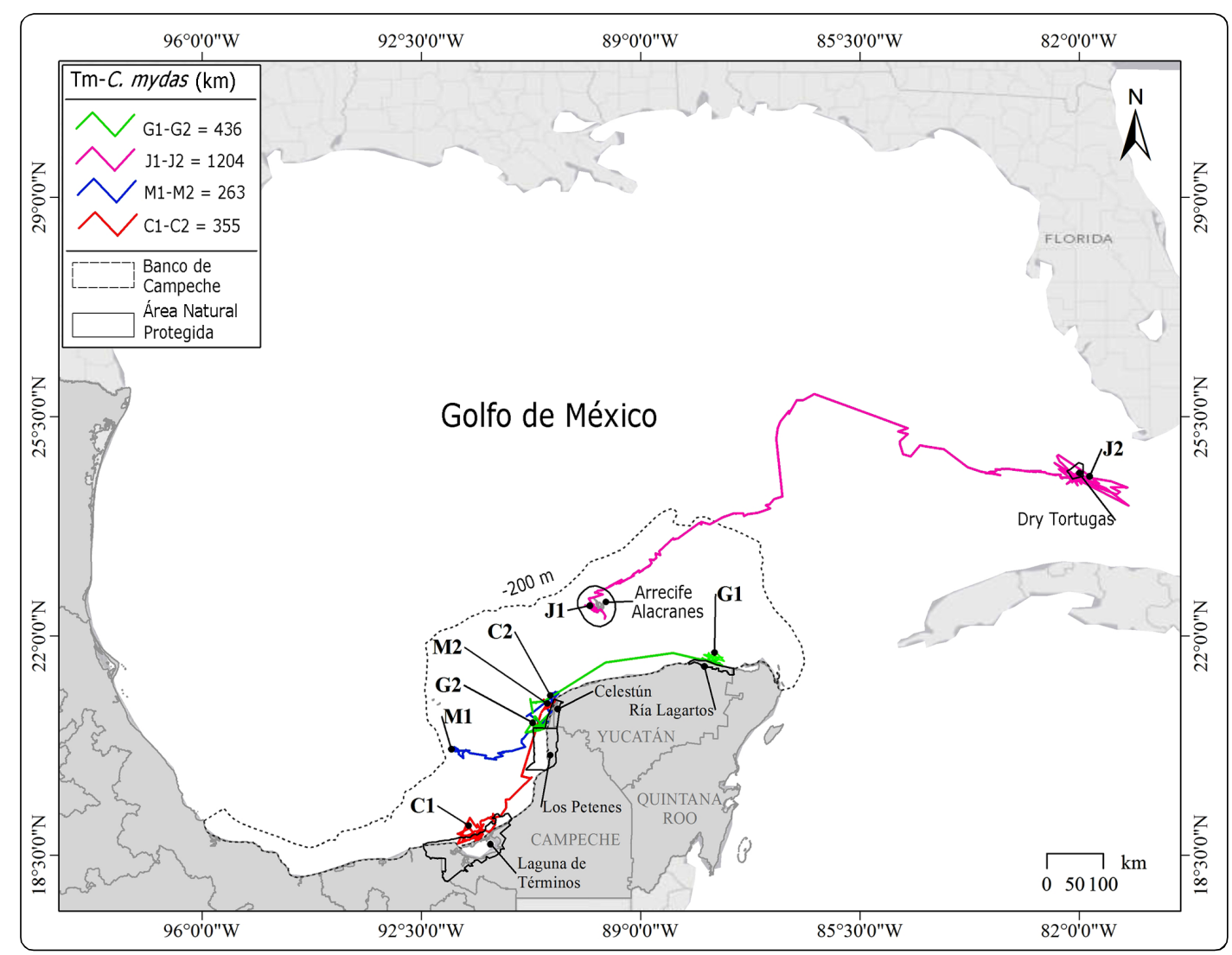

Figura 2. Rutas de migración de 4 hembras post-anidadoras de tortuga C. mydas rastreadas: Gertrudis (G), Julieta (J), Marina (M) y Carmelita (C). Se indican sus puntos iniciales (1) (interanidación) y finales (2) (alimentación) / Migration routes of 4 tracked post-nesting C. mydas females showing their initial (1) (between nesting) and final (2) (feeding) points. Movements of the 4 females tracked are included: Gertrudis (G), Julieta (J), Marina (M) and Carmelita (C)

\section{Resultados}

\section{RUTAS DE MIGRACIÓN}

Las trayectorias migratorias de los 4 individuos adultos de Chelonia mydas post-anidadoras desde sus ámbitos de hogar interanidatorio (1) y de alimentación (2), además de las distancias recorridas, fueron variables (Fig. 2, Tabla 1).

Tres de los 4 individuos de C. mydas post-anidadoras ( G, M y C) establecieron sus ámbitos de hogar (interanidatorios y de alimentación) adyacentes a playas continentales e insulares en la península de Yucatán, a diferencia de $(\mathrm{J})$ que interanidó en territorio mexicano (Arrecife Alacranes) y luego migró a su ámbito de hogar de alimentación en el Parque Nacional Dry Tortugas (en Florida, USA).
Los individuos $(\mathrm{G})$ y (C) utilizaron corredores migratorios adyacentes a las costas de la península de Yucatán en el Banco de Campeche, a isobatas menores a los $100 \mathrm{~m}$ de profundidad dentro de Áreas Naturales Protegidas de México (Reservas de la Biósfera Ría Celestún y Los Petenes, Área de Protección de Flora y Fauna Laguna de Términos y el Parque Nacional Arrecife Alacranes), y una $(\mathrm{J})$ en USA (Parque Nacional Dry Tortugas) (Fig. 2).

La distancia promedio $(\mathrm{km})$ de las localidades de trayectoria a la costa, entre G1 y G2, fue de 15,0 $\pm 3,1 \mathrm{~km}$ (Fig. 2) y de 23,3 $\pm 8,7$ km (entre C1 y C2) para Carmelita.

Las trayectorias migratorias (interanidatorio y de alimentación) de Marina y Julieta fueron las más distantes a la línea de costa donde anidaron. Marina realizó su 
Tabla 1. Valores descriptivos de la migración y establecimiento de 4 tortugas $C$. mydas hembra rastreadas desde la península de Yucatán, México. Distancia total (Dt) de las trayectorias migratorias, ámbito hogareño interanidatorio (1) y de alimentación (2) por el Polígono M ínimo Convexo (PMC), por Kernel Fijo (50-95\%), y su distancia a la costa / Descriptive values of migration and establishment of 4 C. mydas females turtles tracked from the Yucatán Peninsula, Mexico. Total distance of migratory trajectories, internesting (1) and feeding (2) home ranges by Minimum Convex Polygon (PMC), by Fixed Kernel (50-95\%), and its distance to shore

\begin{tabular}{|c|c|c|c|c|c|c|}
\hline \multirow[t]{2}{*}{ C. mydas } & \multirow[t]{2}{*}{$\begin{array}{c}\text { Fase } \\
\text { Migratoria }\end{array}$} & \multirow[t]{2}{*}{$\begin{array}{l}\mathrm{Dt} \\
(\mathrm{km})\end{array}$} & \multirow[t]{2}{*}{$\begin{array}{l}\mathrm{PMC} \\
\left(\mathrm{km}^{2}\right)\end{array}$} & \multicolumn{2}{|c|}{$\begin{array}{c}\text { Kernel Fijo } \\
\left(\mathrm{km}^{2}\right)\end{array}$} & \multirow[t]{2}{*}{$\begin{array}{l}\text { Distancia a la costa }(\mathrm{km}) \\
\quad(\text { Promedio } \pm \mathrm{DE})\end{array}$} \\
\hline & & & & $50 \%$ & $95 \%$ & \\
\hline \multirow[b]{2}{*}{ (0) } & G-1 & 475 & 145 & 15 & 110 & $1,0 \pm 1,2$ \\
\hline 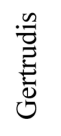 & Migración & 436 & * & $*$ & $*$ & $15,0 \pm 3,1$ \\
\hline & G-2 & 304 & 719 & 76 & 738 & $12,9 \pm 9,0$ \\
\hline \multirow{5}{*}{$\stackrel{\frac{\pi}{0}}{\stackrel{\Xi}{\Xi}} \subseteq$} & Total & 1215 & & & & \\
\hline & $\mathrm{J}-1$ & 116 & 26 & 3 & 27 & $3,6 \pm 1,2$ \\
\hline & Migración & 1204 & $*$ & $*$ & $*$ & * \\
\hline & $\mathrm{J}-2$ & 606 & 65 & 2 & 24 & $4,8 \pm 1,4$ \\
\hline & Total & 1926 & & & & \\
\hline \multirow{3}{*}{ 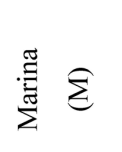 } & M-1 & 140 & 39 & 4 & 33 & $1,0 \pm 1,0$ \\
\hline & Migración & 263 & * & $*$ & $*$ & $*$ \\
\hline & M-2 & 350 & 67 & 6 & 46 & $7,1 \pm 1,4$ \\
\hline \multirow{5}{*}{ 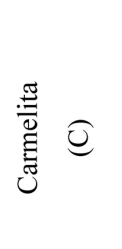 } & Total & 753 & & & & \\
\hline & $\mathrm{C}-1$ & 645 & 1918 & 42 & 282 & $7,3 \pm 7,9$ \\
\hline & Migración & 355 & * & $*$ & * & $23,3 \pm 8,7$ \\
\hline & $\mathrm{C}-2$ & 72 & 322 & 90 & 692 & $7,6 \pm 4,8$ \\
\hline & Total & 1072 & & & & \\
\hline
\end{tabular}

trayectoria migratoria sobre el Banco de Campeche (a isóbatas menores a los $200 \mathrm{~m}$ ) y la segunda (J), sobre el Canal del Golfo México por donde ingresa la Corriente de Yucatán a isóbatas mayores a los 1,000 m.

\section{Clasificación de localidades geográficas}

La tortuga blanca $(\mathrm{G})$ registró en total 528 localidades, de las cuales se utilizaron 235 (45\%); 901 localidades fueron registradas para $(\mathrm{J})$ de las cuáles se utilizaron $757(84 \%)$; (M) registró 551 localidades de las cuales 480 (87\%) fueron utilizadas. Finalmente, fueron registradas 253 localidades para (C) y se utilizaron 202 (80\%).

El promedio en días del rastreo satelital de las 4 tortugas blancas fue de $123 \pm 58$. El rango porcentual sumado del tiempo de permanencia registrado dentro de los ámbitos de hogar (interanidatorio y de alimentación) de los 4 individuos post-anidadores fue de $89-93 \%$, el restante 7 $11 \%$ del tiempo fue utilizado por las tortugas para desplazarse entre ámbitos de hogar (trayectoria migratoria o migración).
Las trayectorias descritas dentro de los límites de ámbitos de hogar delimitados por el PMC para (G) durante su interanidación fue de $475 \mathrm{~km}$ y $304 \mathrm{~km}$ durante su alimentación (Fig. 3); para (J) en su interanidación y alimentación fue de 116 y $606 \mathrm{~km}$ respectivamente; 140 $\mathrm{km}$ y $350 \mathrm{~km}$ para (M) en su interanidación y alimentación. Finalmente, (C) se desplazó 645 km y 72 km en sus ámbitos hogareños de interanidación y alimentación. Finalmente, la tortuga blanca (C), se desplazó en C1 y C2, 645 km y 72 $\mathrm{km}$ respetivamente dentro de sus ámbitos de hogar inicial y final.

Trayectorias Migratorias, ÁMbitos de hogar INTERANIDATORIOS Y DE ALIMENTACIÓN

La distancia promedio total de desplazamiento migratorio por las 4 tortugas blancas fue de $1242 \pm 496 \mathrm{~km}$. Las distancias promedio entre localidades registradas por las 4 tortugas blancas post-anidadoras dentro de los ámbitos de hogar en zonas marinas continentales fue de $0,7 \pm 2.4$ $\mathrm{km}$ y de $0,5 \pm 1.0 \mathrm{~km}$ en zonas marinas insulares. 


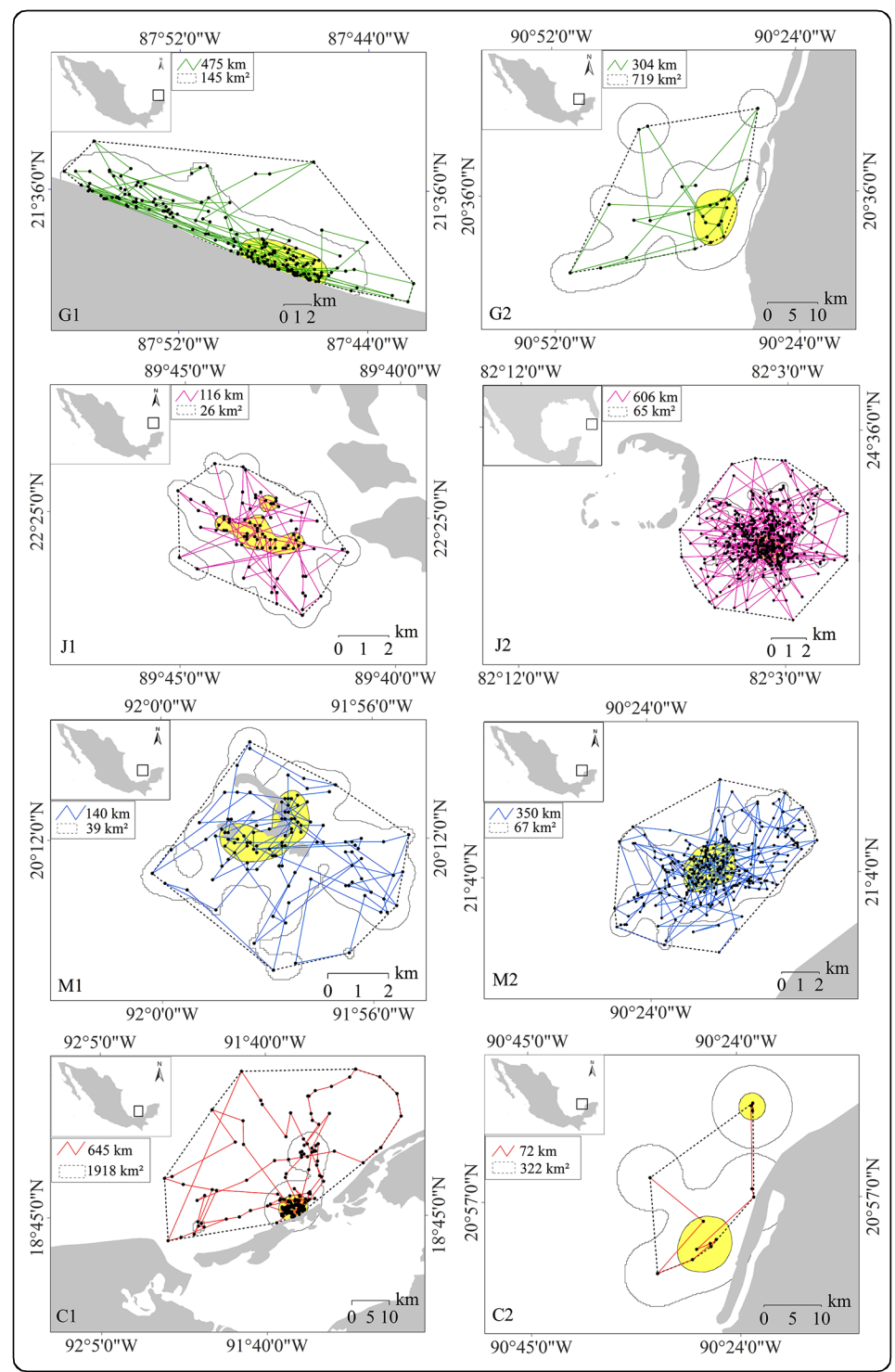

Figura 3. Mapas de los movimientos dentro de los ámbitos de hogar interanidatorio (1) y de alimentación (2) para 4 individuos de C. mydas post-anidadoras (G: Gertrudis, J: Julieta, M: Marina, y C: Carmelita), rastreadas desde la península de Yucatán, México / Movements inside internesting (1) and feeding (2) home ranges by 4 post nesting C. mydas individuals (G: Gertrudis, J: Julieta, M: Marina, and C: Carmelita), tracked from the Yucatán Peninsula, Mexico
Los individuos de C. mydas (G), (M) y (C) coincidieron con su ámbito de hogar de alimentación en Celestún y Los Petenes superponiéndose entre áreas delimitadas por el PMC en una superficie de $25 \mathrm{~km}^{2}$.

Las áreas abarcadas por los ámbitos de hogar adyacentes a la costa continental (península de Yucatán) e islas, estimados por los métodos de PMC y KF50-95\% para las 4 hembras de C. mydas post-anidadoras fueron significativamente diferentes ( $\mathrm{H}=14,6 ; P<0,01 ; \alpha=0,05)$ (Fig. 4).

También se aprecian diferencias significativas entre las áreas delimitadas por el PMC adyacentes al continente respecto de las áreas de KF50\% adyacentes de islas, y de esta última con áreas de KF95\% adyacentes también a islas (Fig. 5).

\section{Distancia de las localidades a la costa}

La distancia promedio de los ámbitos hogareños a la costa de los individuos que establecieron sus áreas cerca de las costas continentales fue de 5,7 $\pm 5,4 \mathrm{~km}$, distancia significativamente mayor que la distancia promedio de las tortugas blancas que establecieron sus ámbitos de hogar cerca de las costas insulares 4,0 $\pm 1,9 \mathrm{~km}(\mathrm{H}=38,0$; $P<0,05 ; \alpha=0,05)$.

Los ámbitos de hogar (interanidatorio y de alimentación) pueden estar adyacentes a las costas continentales e insulares, por lo general distantes y de mayor área en el primer caso respecto al segundo. Dentro de los ámbitos de hogar interanidatorio y de alimentación se evidencian además centros de mayor y menor actividad. 


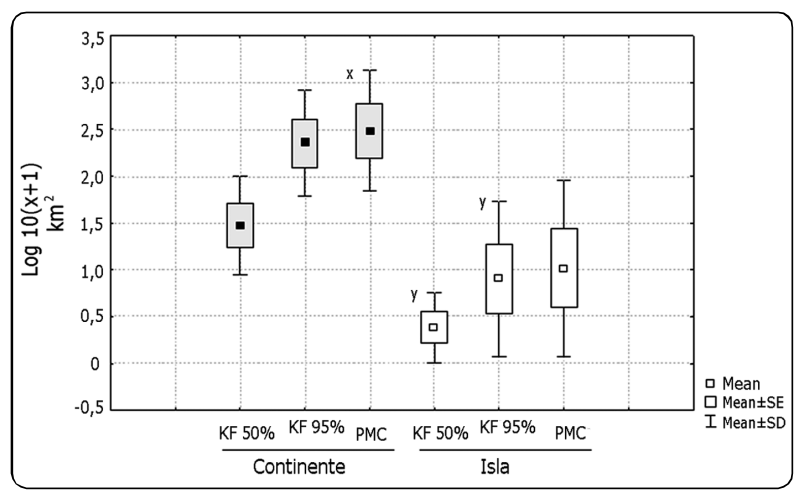

Figura 4. Gráfico de cajas y bigotes de comparación de superficies de ámbitos hogareños interanidatorios y de alimentación (PMC y KF) de 4 individuos de $C$. mydas post-anidadoras, con base en la prueba de Dunn $(H=14,6 ; P<0,05, \alpha=0,05)$. La diferencia en letras $(x, y)$ indican una correspondencia significativa entre medianas / Comparative box and whiskers graph for internesting and feeding home range surfaces for $4 \mathrm{C}$. mydas post-nesting females, based on a Dunn test $(H=14.6 ; P<0.05, \alpha=0.05)$. Different letters $(x, y)$ indicate a significant correspondence between medians

\section{NaVEGaCión y RUMbo}

En la figura 5 se presentan los registros de los rumbos y acimut que describieron las 4 tortugas rastreadas durante sus trayectorias migratorias, desde el ámbito de hogar inicial (interanidación) hasta ámbitos de hogar de alimentación (ámbito de hogar final).De manera general, se observó un rumbo definido para el movimiento de las hembras entre sus hábitats de internadicación y alimentación. Dicho movimiento con un rumbo definido y constante, sin variaciones significativas en su navegación, muestra la capacidad de navegación y orientación para moverse por cientos de kilómetros en mar abierto hacia un punto particular a priori que ellas definen con base en su experiencia y necesidades.

\section{Discusión}

\section{RUtas Migratorias}

Dado el uso como hábitat de alimentación y migración de las aguas adyacentes a la península de Yucatán de al menos 4 especies diferentes de tortugas marinas (Cuevas et al. 2008), se sugiere que dichas poblaciones simpátricas comparten sus hábitats críticos marinos en alguna proporción, y la información sobre ámbitos de hogar y patrones de migración obtenida para una especie de tortuga marina, podría ser de utilidad para las otras especies también (Mortimer \& Portier 1989, Cuevas et al. 2008, Marcovaldi et al. 2010).
Durante la temporada de anidación, los 4 individuos de C. mydas post-anidadores estudiados) evidenciaron ámbitos de hogar interanidatorios y de alimentación, ocupando entre un 89 y $93 \%$ de su tiempo para esta actividad y el restante 7 y $11 \%$ del tiempo lo utilizan para migrar entre ámbitos de hogar.

Tröeng et al. (2005) encontraron comportamientos similares con el rastreo satelital de 10 tortugas hembras adultas de C. mydas en Tortuguero, Costa Rica, evidenciando rutas migratorias y zonas de alimentación e interanidación adyacentes a las costas. Por su parte, Hart \& Fujisaki (2010) encontraron con el rastreo de 6 individuos juveniles de C. mydas desde la costa sudoeste de la península de Florida, evidencia del establecimiento de ámbitos de hogar de alimentación cercanos a la costa.

MacDonald et al. (2012) reportaron en su estudio realizado en el rastreo de 25 individuos de $C$. mydas (machos y hembras), un comportamiento de establecimiento de áreas de ámbitos de hogar de

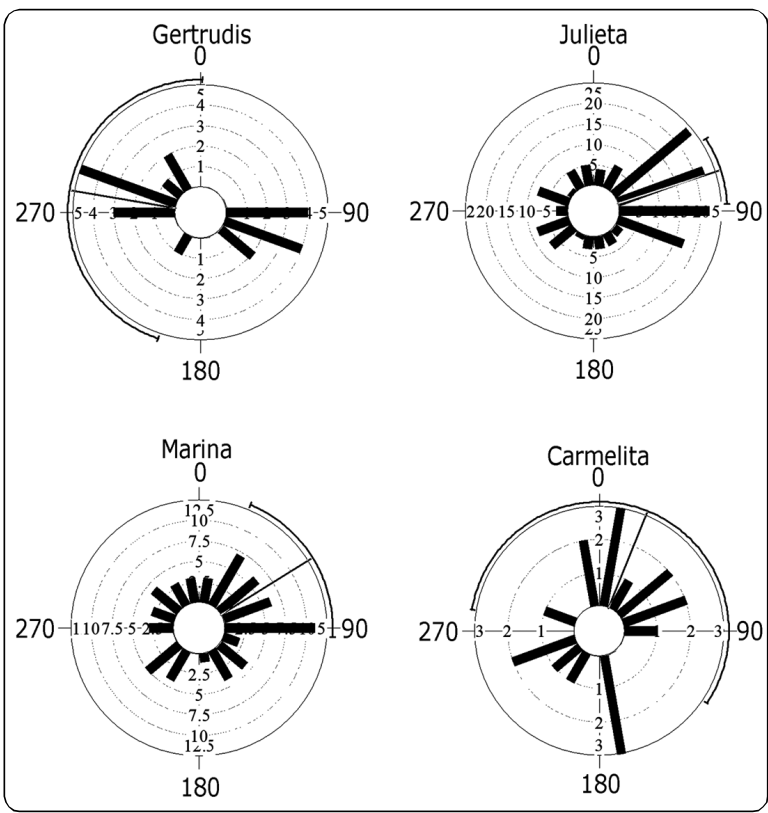

Figura 5. Histogramas circulares del rumbo de navegación de las trayectorias migratorias de $4 \mathrm{C}$. mydas post-anidadoras desde sus playas de anidación a sus sitios de alimentación. La línea solida curva representa la desviación estándar y el eje perpendicular conectado a ésta (línea sólida delgada), indica el acimut de orientación promedio del desplazamiento / Circular histograms of the navigation heading during migratory routes by 4 post nesting $C$. mydas from their nesting beaches to their feeding places. Solid curve line represents standard deviation and the perpendicular axe connected to this line indicates the average movement orientation azimuth 
alimentación en la zona marina de San Diego Bay, California, USA.

En este estudio, las tortugas más pequeñas, Julieta y Gertrudis se desplazaron distancias mayores de (1,204 y $436 \mathrm{~km}$ respectivamente) entre ámbitos de hogar interanidatorio y de alimentación, mientras que Marina y Carmelita de mayor talla se desplazaron distancias menores (110 y $112 \mathrm{~km}$ respectivamente) entre sus ámbitos de hogar interanidatorio y de alimentación.

La mayor distancia de trayectoria migratoria registrada para C. mydas superó los $2.496 \mathrm{~km}$ entre la Isla Ascención y Brasil (Seminoff et al. 2008, Hays et al. 2002). Distancias migratorias largas suponen poblaciones de mayor distribución en hábitats críticos marinos, y distancias cortas una distribución más localizada para especies como E. imbircata, Lepidochelys olivacea, Lepidochelys kempii (Meylan 1999, Proietti et al. 2009).

En la península de Yucatán, en los ámbitos de hogar para C. mydas estimados por el PMC, hubo una tendencia de mayor área $\left(629 \pm 766 \mathrm{~km}^{2}\right)$ en la zona marina insular continental respecto a las áreas menores de los ámbitos de hogar insulares $\left(53 \pm 23 \mathrm{~km}^{2}\right)$. También se evidenciaron mayores distancias entre áreas de ámbitos de hogar con la costa continental peninsular $(5,7 \pm 5,4 \mathrm{~km})$, mientras en costas insulares, las áreas de ámbitos de hogar estaban a menor distancia $(4,0 \pm 1,9 \mathrm{~km})$. Esta información sugiere un uso distinto de las áreas marinas aledañas a sus playas de anidación entre zonas continentales e insulares, pudiéndose relacionar con la disponibilidad de refugio y alimento (Hays et al. 2002, Van Dam et al. 2008).

Cuevas et al. (2008) reportaron en áreas marinas de la península de Yucatán para un individuo de E. imbricata post-anidador rastreado satelitalmente, una distancia menor a los $10 \mathrm{~km}$ entre ámbitos de hogar con la costa insular (Isla Mujeres). Mientras que la distancia entre el ámbito de hogar y la costa continental para un segundo individuo post-anidador de la misma especie fue menor a los $70 \mathrm{~km}$. Estos mismos autores, en su investigación sobre E. imbricata post-anidadoras en la misma región que el presente estudio, reportaron superficies de ámbitos de hogar similares (en un rango de 88 a $618 \mathrm{~km}^{2}$ ) al reportado en este estudio para $C$. mydas. Los autores señalan además que ámbitos de hogar de superficies menores respecto a los de mayor superficie, podrían estar relacionados a la disponibilidad de recursos alimenticios.

Existen reportes de individuos juveniles de C. mydas con ámbitos de hogar en un rango de 3.741 a $2.060 \mathrm{~km}^{2}$ adyacentes a la costa de la península de Florida, lo que hace suponer que tal diferencia en el área abarcada por juveniles y adultos de la misma especie representa un uso más diversificado de sus áreas de estancia por parte de los primeros (Hart \& Fujisaki 2010).

Por su parte Senko et al. (2010) reportaron en promedio 4,040 $\pm 3,860$ (DE) $\mathrm{km}^{2}$ de superficie de ámbitos de hogar para juveniles de tortuga blanca en la zona marina nerítica de la Península de California.

Kinzel et al. (2003) reportó ámbitos hogareños de alimentación (Polígono Mínimo Convexo) de entre 335 y $2740 \mathrm{~km}^{2}$ para tortugas hembra de $C$. mydas, y de entre 220 y $360 \mathrm{~km}^{2}$ para la zona de mayor densidad de ubicaciones por el método Kernel. Estos valores son mayores que los registrados en este estudio, lo que podría deberse a errores de las ubicaciones geográficas de los transmisores tal y como lo sugieren los mismos autores.

Renaud \& Carpenter (1994) reportaron un rango de ámbito de hogar de 954 a 4,279 km² para Caretta caretta en la zona marina poco profunda al norte del Golfo de México. Es evidente que Caretta caretta ocupó ámbitos de hogar de mayor área respecto a $C$. mydas y $E$. imbricata. Van Dam \& Diez (1996), León \& Bjorndal (2002) señalaron que las diferencias entre el tamaño de las superficies de áreas de ámbitos de hogar, de diferentes especies de tortugas marinas, podrían estar relacionadas con requerimientos de ámbitos de hogar diferentes que demandan durante la etapa post-anidatoria.

Por otra parte, los resultados sugieren que C. mydas post-anidadora utiliza corredores migratorios en la plataforma marina del Banco de Campeche para trasladarse entre áreas de descanso y alimentación. Pero también tiene la capacidad de trasladarse grandes distancias ocupando áreas marinas en territorio de otros países para dichas actividades, como fue el caso de Julieta, que se traslado $1.204 \mathrm{~km}$ entre su ámbito de hogar interanidatorio (J1) en Arrecife Alacranes hasta su ámbito de hogar de alimentación (J2) (en Dry Turtles) ubicado en la plataforma marina de la península de Florida (USA).

Hays et al. (2002) reportaron distancias migratorias para C. mydas post-anidadoras mayores a los $2.000 \mathrm{~km}$, y más de $60 \mathrm{~km}$ al día. En nuestro estudio C. mydas se desplazó en promedio $564 \pm 432 \mathrm{~km}$ de distancia entre ámbitos de hogar interanidatorio y de alimentación a 48,7 $\pm 24,9 \mathrm{~km} \mathrm{día}^{-1}$. Lo que evidencia que $C$. mydas tiene gran capacidad para migrar y ocupar hábitats críticos compartidos entre naciones de una misma región, como lo reportaron Moncada et al. (2006) a través de datos de marca y recaptura de hembras anidadoras. 
Debido a la gran capacidad migratoria de algunas especies de tortugas marinas, entre ellas C. mydas y la importancia de áreas marinas cercanas entre sí compartidas entre países que integran el Gran Caribe, Blumenthal et al. (2006) y Seminof et al. (2008) recomiendan que los esfuerzos de conservación para estos quelonios debieran fortalecerse a escalas internacionales. Colonias de tortugas marinas que comparten corredores migratorios y áreas de descanso y alimentación, son vulnerables o favorecidas, por las diferentes políticas y realidades socio-económicas de cada país (CIT 2012).

En esta investigación 3 de las 4 tortugas blancas postanidadoras rastreadas ( $\mathrm{G}, \mathrm{C}$ y $\mathrm{M}$ ) atravesaron $\mathrm{y}$ establecieron sus ámbitos de hogar (interanidatorio y de alimentación) en zonas neríticas marinas de la península de Yucatán.

\section{Navegación de Chelonia MYDAS}

Marcovaldi et al. (2010), Mortimer and Portier (1989) reportaron que los individuos post-anidadores de tortugas marinas tienen un patrón de migración adyacentes a la línea de la costa entre ámbitos de hogar. En la península de Yucatán, las tortugas blancas rastreadas en este estudio, y carey post-anidadoras (Cuevas et al. 2008) evidenciaron un patrón migratorio por las zonas marinas poco profundas. Ambos resultados respaldan con mayor certeza la presencia de corredores importantes para estas 2 especies de tortugas marinas en la zona nerítica poco profunda.

Por otro lado, las corrientes geostróficas no evidenciaron tener influencia en las tendencias de desplazamiento durante los periodos de migración de $C$. mydas post-anidadoras. Zavala-Hidalgo et al. (2003) y Cuevas et al. (2008) indicaron que en varias regiones oceánicas las corrientes marinas no son un factor que pueda determinar el desplazamiento de las tortugas marinas en periodo de migración, fundamentados en el tamaño, que sugiere independencia en el traslado de los individuos adultos en ambientes marinos pelágicos.

Sin embargo, Walcott et al. (2012) señalan que las corrientes marinas sí podrían ser un factor ambiental a favor de las tortugas marinas que se desplazan largas distancias en mar abierto. La tortuga Julieta (J) mostró una tendencia de asociación a la Corriente de Lazo (después de la isóbata 200 del Banco de Campeche) para atravesar el Canal del Golfo de México y llegar a su ámbito de hogar de alimentación en Dry Tortugas (Florida en USA).
En 1999 Garduño-Andrade et al. reportaron un comportamiento similar de migración de una tortuga $C$. mydas hembra liberada desde Isla Mujeres, México. Esta hembra cruzó el Golfo de México para establecerse en una zona aledaña a la registrada en Florida con el rastreo de hembras en este estudio. Adicionalmente, Kinzel et al. (2003) registraron 2 hembras de $C$. mydas cruzando el Golfo de México con un comportamiento similar a las de este estudio, y estableciéndose de igual forma en el Santuario Marino Nacional de los Cayos de Florida.

Se sugiere que las tortugas marinas post-anidadoras utilizan las corrientes marinas alternativamente cuando tienen la oportunidad, permitiéndoles hacer un uso eficiente de su energía destinada para la migración (Walcott et al. 2012). En el Golfo de México, es probable que algunas de las tortugas blancas post-anidadoras asocian alternativamente sus movimientos migratorios de desplazamientos local (dentro de los límites de la plataforma marina del Banco de Campeche) a las corrientes de origen geostrófico ciclónicos y anticiclónicos, estos últimos originados en la Corriente de Lazo en el Golfo de México.

La Corriente de Yucatán ingresa a aguas del Golfo de México $\left(2.522 \pm 106 \mathrm{~m}^{3} \mathrm{~s}^{-1}\right)$ por el canal del mismo nombre (estimación basada en 10 meses de muestreo). La velocidad de entrada de la Corriente de Lazo es de $9 \mathrm{~km} \mathrm{~h}^{-1}$ aproximadamente (Sheinbaum et al. 2002). La tendencia de la orientación de Julieta en medio del Canal de Golfo, con la orientación de la Corriente de Lazo (en forma de ' $U$ '), sugiere una desviación involuntaria de la tortuga en su recorrido directo a su zona de alimentación, lo cual podría deberse a la deriva causada por cruzar el fuerte flujo de corriente de agua en esta zona del Golfo de México. Una vez que entró a la plataforma de la península de Florida, donde la Corriente de Lazo pierde velocidad, mantuvo nuevamente un rumbo fijo y directo a la zona donde estableció finalmente su ámbito de hogar de alimentación alrededor de la isla Dry Tortugas.

La dinámica de entrada y salida de corrientes en la Cuenca del Golfo, al margen de la importancia que representa para el transporte de nutrientes, posiblemente representa un mecanismo importante de transporte que algunas especies de tortugas marinas post-anidadoras, entre ellas C. mydas, aprovecha para establecerse entre distintos ámbitos de hogar, utilizando la menor energía corporal para transportarse.

Kinzel et al. (2003) reportaron un incremento de la velocidad de nado de la tortuga rastreada al ingresar a la 
Corriente de Lazo, sugiriendo una relación directa con esta característica oceanográfica en la región.

\section{Conflictos CON LA CONSERVACIÓN DE LA ESPECIE}

El conocimiento del comportamiento de los movimientos de tortugas marinas es importante dentro de los programas de gestión y manejo costero marino, debido a los roles ecológicos y económicos que se pueden generar con poblaciones estables de las especies de tortugas presentes a la región, beneficiando a los ambientes marinos donde habitan peces de importancia comercial y que podrían ser además considerados dentro de programas de turismo (Troëng \& Drews 2004).

Identificar y localizar los hábitats utilizados por las tortugas marinas es útil para definir áreas prioritarias de conservación para su protección, asegurando que cumplan con sus funciones ecológicas y generen beneficios económicos locales y/o regionales.

Las tortugas marinas son particularmente vulnerables a capturas incidentales durante la etapa migratoria, debido a la actividad pesquera (Wallace et al. 2010). Las costas de la península de Yucatán, se han constituido desde hace más de 5 décadas como zonas de pesca de especies comerciales importante para la economía local, regional y nacional, actividad realizada por flotas pesqueras artesanales e industriales (Mexicano-Cintora et al. 2009, Aguilar \& Tuz 2010).

La flota artesanal generalmente opera cerca de las costas (a isóbatas menores a los $100 \mathrm{~m}$ ), y a isóbatas mayores a los $100 \mathrm{~m}$, son las operaciones de la flota industrial (Salas et al. 2006). En ésta investigación las áreas de ámbitos de hogar de C. mydas post-anidadoras fueron registradas a isóbatas menores a $\operatorname{los} 100 \mathrm{~m}$, lo que sugiere que las poblaciones de tortugas blancas de esta cohorte, actual e históricamente estuvieron expuestas a la pesca incidental artesanal al igual que E. imbricata. Cuevas et al. (2008) identificaron también áreas de ámbitos de hogar y rutas migratorias para E. imbricata en zonas neríticas de la península de Yucatán. Los resultados expuestos en este trabajo de investigación son de relevante importancia para determinar nuevos retos en la planificación de áreas de conservación para las especies de tortugas marinas presentes en la península de Yucatán.

\section{Agradecimientos}

Los autores agradecen al Fondo Sectorial de Investigación CONACYT-SEMARNAT por el financiamiento del proyecto $\mathrm{N}^{\mathrm{o}} 107770$ en el cual se dio esta investigación. A la Cooperación Técnica Belga en Bolivia, por financiar los estudios de posgrado, que dieron como resultado esta publicación del primer autor. A la Dirección General de Vida Silvestre de la SEMARNAT por el permiso de colecta científica SGPA/DGVS/04530/11 otorgado para la realización de este proyecto. A la Dirección del Parque Nacional Arrecife Alacranes, Reserva de la Biosfera Ría Lagartos y Área de Protección de Flora y Fauna Laguna de Términos, en particular a Patricia Huerta-Rodríguez Pedro García-Alvarado, de la Comisión Nacional de Áreas Naturales Protegidas por el apoyo brindado para la colocación de los transmisores satelitales. A la Secretaría de Marina Armada de México, Estación de Investigación Oceanográfica del Carmen, Tercera Región Naval, en especial al Capitán Roberto Flores Rodríguez y al Teniente Edwalt Hernández Torralba, por el apoyo crítico y decidido para la colocación de un transmisor satelital en Cayo Arcas. A los Doctores Alfonso Aguilar y Silvia Hernández por dar seguimiento al manuscrito. Y a los revisores por sus valiosas aportaciones para completar este manuscrito.

\section{LITERATURA CITADA}

Aguilar A \& A Tuz. 2010. Pesca, manejo y conservación del negrillo (Mycteroperca bonaci) en la costa norte de la Península de Yucatán. Bioagrociencias 3(1):35-37.

ARGOS. 1996. User's Manual. CLS/Service Argos, Toulouse. $<\mathrm{http} / / /$ www.argos-system.org/manual>

Balazs GH, RK Miya \& SC Beavers. 1996. Procedures to attach a satellite transmitter to the carapace of an adult green turtle, Chelonia mydas. In: Keinath JA, DE Barnard JA Musick \& BA Bell (eds). Proceedings of the 15th Annual Symposium on Sea Turtle Biology and Conservation NMFS-SEFSC-387: 21-26.

Blumenthal JM, JL Solomon, CD Bell, TJ Austin, G Ebanks-Petrie, MS Coyne, AC Broderick \& BJ Godley. 2006. Satellite tracking highlights the need for international cooperation in marine turtle management. Endangered Species Research 7: 1-11.

Bowen BW, AB Meylan, P Ross, CJ Limpus, GH Balazs \& JC Avise. 1992. Global population structure and natural history of the green turtle (Chelonia mydas) in terms of matriarchal phylogeny. Evolution 46: 865-881.

Byles RA \& YB Swimmer. 1994. Post-nesting migration of Eretmochelys imbricata from the Yucatan Peninsula. In: Bjorndal KA, AB Bolten, DA Jhonson \& PJ Eliazar (eds). Proceedings of the 14th Annual Symposium on Sea Turtle Biology and Conservation NMFS-SEFSC-351: 202.

CIT. 2012. Estado de conservación y uso de hábitats de las tortugas marinas en el océano pacífico oriental. CIT-CC82011-Tec.1: 1-27. 
Coyne MS \& BJ Godley. 2005. Satellite tracking and analysis tool (STAT): an integrated system for archiving, analyzing and mapping animal tracking data. Marine Ecology Progress Series 301: 1-7.

Cuevas E, MA Liceaga-Correa \& M Garduño-Andrade. 2007. Spatial characterization of a foraging area for immature hawksbill turtles (Eretmochelys imbricata) in Yucatan, Mexico. Amphiban-Reptilia 28: 337-346.

Cuevas E, FA Abreu-Grobois, V Guzmán-Hernández, MA Liceaga-Correa \& RP Van Damme. 2008. Post-nesting migratory movements of hawksbill turtles Eretmochelys imbricata in waters adjacent to the Yucatan Peninsula, Mexico. Endangered Species Research: 1-12. <doi: 10.3354/ esr00128>

Day RW \& GP Quinn. 1989. Comparisons of treatments after an analysis of variance in ecology. Ecological Monographs 59(4): 433-463.

Eckert KL \& FA Abreu-Grobois. 2001. Conservación de tortugas marinas en la Región del Gran Caribe - un diálogo para el manejo regional efectivo, $170 \mathrm{pp}$. WIDECAST, UICN/CSE Grupo Especialista en Tortugas Marinas (MTSG), WWF y el Programa Ambiental del Caribe del PNUMA, St. Croix, U. S. Virgin Islands. [Traducción al español por R Briseño Dueñas y F Alberto Abreu Grobois]

Fancy SG, LF Pank, DC Douglas, CH Curby, GW Garner, SC Amstrup \& WL Regelin. 1988. Satellite telemetry: A new tool for wildlife research and management, 84 pp. Fish and Wildlife Service, Alaska Fish and Wildlife Research Center, Washington.

Garduño M, A Maldonado, R Márquez, B Schroeder \& G Balazs. 2000. Satellite tracking of an adult male and female green turtle from Yucatan in the Gulf of Mexico. In: Kalb HJ \& T Wibbels (Comps). Proceedings of the Nineteenth Annual Symposium on Sea Turtle Biology and Conservation. U. S. Dept. Commerce. NOAA Technical Memorandum, NMFS-SEFSC-443: 158-159.

Garduño M, R Márquez, B Schroeder \& G Balazs. 2000a. Migración y buceo de la tortuga Carey en la Península de Yucatán. In: Memorias del X Taller y I Congreso Regional sobre Programas de Conservación de Tortugas Marinas en la Península de Yucatán. [CD ROM].

Garduño M, A Maldonado \& R Lope. 2000b. Dinámica poblacional de la tortuga de carey (Eretmochelys imbricata) en su área de forrajeo, Río Lagartos, Yucatán, 60 pp. CONABIO, México.

Groombridge B. 1982. The IUCN Amphibian-Reptilia red data book, part 1. Testudines Crocodylia Rhynchocephalia, 426 pp. IUCN, Gland.

Groombridge B \& R Luxmoore. 1989. The green turtle and hawksbill (Reptilia: Cheloniidae) world status, exploitation and trade, 573 pp. IUCN Conservation Monitoring Centre, Cambridge.

Hart KM \& I Fujisaki. 2010. Satellite tracking reveals habitat use by juvenile green sea turtles Chelonia mydas in the Everglades, Florida, USA. Endangered Species Research 11: 221-232.
Hart KM, MM Lamont, I Fujisaki, AD Tucker \& RR Carthy. 2012. Common coastal foraging areas for loggerheads in the Gulf of Mexico: Opportunities for marine conservation. The Biological Conservation 145: 185-194.

Hays CG, AC Broderick, BJ Godley, P Lovell, C Martin, BJ McConnell \& S Richardson. 2002. Biphasal long-distance migration in green turtles. Animal Behaviour 64: 895-898.

Hooge PH, WM Eichenlaub \& EK Solomon. 2000. Using GIS to analyze animal movements in the marine environment, 20 pp. United States Geological Survey, Alaska Biological Science Center, Gustavus.

James MC, CA Ottensmeyer \& RA Myers. 2005. Identification of high-use habitat and threats to leatherback sea turtles in northern waters: new directions for conservation. Ecology Letters 8: 195-201.

Kinzel M, G Carter, G Tiburcio-Pintos \& R Bravo-Gamboa. 2003. Home range and habitat analysis of green sea turtles, Chelonia mydas, in the Gulf of Mexico. In: Seminoff JA (Comp). Proceedings of the Twenty-Second Annual symposium on Sea Turtle Biology and Conservation. NOAA Technical Memorandum, NMFS-SEFSC-503: 289290.

León YM \& KA Bjorndal. 2002. Selective feeding in the hawksbill turtle, an important predator in coral reef ecosystems. Marine Ecology Progress Series 245: 249-258.

Lewison RL \& LB Crowder. 2007. Putting longline bycatch of Sea Turtles into perspective. Conservation Biology 21(1): 79-86.

Lohmann KJ, CMF Lohmann \& NF Putman. 2007. Magnetic maps in animals nature's GPS. The Journal of Experimental Biology 210: 3697-3705.

MacDonald BD, RL Lewison, SV Madrak, JA Seminoff \& T Eguchi. 2012. Home ranges of East Pacific green turtles Chelonia mydas in a highly urbanized temperate foraging ground. Marine Ecology Progress Series 461: 211-221.

McClenachan L, JBC Jackson \& MJH Newman. 2006. Conservation implications of historic sea turtle nesting beach loss. Frontiers in Ecology and the Environment 4(6): 290-296.

Mexicano-Cintora G, MA Liceaga-Correa \& S Salas. 2009. Usos de sistemas de información geográfica en pesquerías: la pesca en Yucatán, al sur del Golfo de México. Universidad y Ciencia 25(1): 23-38.

Marcovaldi MA, GG Lopez, LS Soares, E Lima, J Thomé \& A Almeida. 2010. Satellite tracking of female loggerhead turtles highlights fidelity behavior in northeastern Brazil. Endangered Species Research 12: 263-272.

Márquez R. 1996. Las tortugas marinas y nuestro tiempo, 197 pp. Fondo de Cultura Económica, México, México. <http://www.bionica.info/biblioteca/Marquez1996LasTortugasMarinas.pdf>

Meylan A. 1999. International movements of immature and adult Hawksbill turtles (Eretmochelys imbricata) in the Caribbean Region. Chelonian Conservation Biology 2: 189194. 
Moncada F, FA Abreu-Grobois, A Muhlia-Melo, C Bell, S Tröeng, KA Bjorndal, AB Bolten, AB Meylan, J Zurita, G Espinosa, G Nodarse, R Márquez-Millán, A Foley \& L Ehrhart. 2006. Movement patterns of green turtle (Chelonia mydas) in Cuba and adjacent Caribbean waters inferred from flipper tag recaptures. Journal of Herpetology 1: 22-34.

Mortimer JA \& KM Portier. 1989. Reproductive homing and interesting behaviour of the green turtle (Chelonia mydas) at Ascension Island, South Atlantic Ocean. Copeia 1989: 962-977.

Peare T \& PG Parquer. 1996. Local genetic structure within two rookeries of Chelonia mydas (The green turtle). Heredity 77: 619-628.

Pritchard PCH \& JA Mortimer. 1999. Taxonomía, morfología externa e identificación de las especies. En: Eckert KL, KA Bjorndal \& FA Abreu-Grobois \& M Donnelly (eds). Técnicas de Investigación y manejo para la conservación de las tortugas marinas 4: 23-41. UICN/SCE Grupo especialista en tortugas marinas, Pensilvania.

Proietti MC, P Lara-Ruiz, JW Reisser, LS Pinto, OA Dellagostin \& LF Marins. 2009. Green turtles (Chelonia mydas) foraging at Arvoredo Island in Southern Brazil: Genetic characterization and mixed stock analysis through mtDNA control region haplotypes. Genetics and Molecular Biology 32(3): 613-618.

Renaud ML \& JA Carpenter. 1994. Movements and submergence patterns of loggerhead turtles (Caretta caretta) in the Gulf of Mexico determined through satellite telemetry. Bulletin of Marine Science 55(1): 1-15.

Salas S, G Mexicano-Cíntora \& MA Cabrera. 2006. ¿Hacia dónde van las pesquerías en Yucatán? Tendencias, retos y perspectivas, 97 pp. Centro de Investigación y de Estudios Avanzados del Instituto Politécnico Nacional, Mérida.

Seminoff JA. 2004. Chelonia mydas. In: IUCN (ed). IUCN Red List of Threatened Species. Version 2013.2. Southwest Fisheries Science Center, IUCN. <http:// www.iucnredlist.org/details/4615/0>.

Seminoff AJ, P Zárate, M Coyne, DG Foley, D Parker, BN Lyon \& PH Dutton. 2008. Post-nesting migrations of Galápagos green turtles Chelonia mydas in relation to oceanographic conditions: integrating satellite telemetry with remotely sensed ocean data. Endangered Species Research 4: 57-72.
Senko J, V Koch, WM Megill, RR Carthy, RP Templeton \& WJ Nichols. 2010. Fine scale daily movements and habitat use of East Pacic green turtles at a shallow coastal lagoon in Baja California Sur, Mexico. Journal of Experimental Marine Biology and Ecology 391: 92-100.

Sheinbaum J, J Candela, A Badan \& J Ochoa. 2002. Flow structure and transport in the Yucatan Channel. Geophysical Research Letters 29(3): 1040.

Troëng S \& C Drews. 2004. Money talks: economic aspects of marine turtle use and conservation, $64 \mathrm{pp}$. WWFInternational, Gland.

Troëng S, DR Evans, E Harrison \& CJ Lagueux. 2005. Migration of green turtles Chelonia mydas from Tortuguero Costa Rica. Marine Biology 148: 435-447.

Van Dam RP \& CE Diez. 1996. Predation by hawksbill turtles on sponges at Mona Island, Puerto Rico. In: Lessios HA \& IG Macintyre (eds). Proceedings of the 8th International Coral Reef Symposium 2: 1421-1426.

Van Dam RP, CE Diez, GH Balaz, LA Colón-Colón, WO McMillan \& B Schoerder. 2008. Sex-specific migration patterns of hawksbill turtles breeding at Mona Island, Puerto Rico. Endangered Species Research 3: 85-94.

Walcott J, S Eckert \& JA Horrocks. 2012. Tracking hawksbill sea turtles (Eretmochelys imbricata) during inter-nesting intervals around Barbados. Marine Biology 159: 927-983.

Wallace BP, RL Lewison, SL McDonald, CY Kot, S Kelez, RK Bjorkland, EM Finkbeiner, S Helmbrecht \& LB Crowder. 2010. Global patterns of marine turtle bycatch. Conservation Letters 3(3): 131-142.

Witt MJ \& EA Bonguno, AC Broderick, MS Coyne, A Formia, A Gibudi, GA Mounguengui, C Moussounda, M Nsafou, S Nougessono, RJ Parnell, GP Sounguet, S Verhade \& BJ Godley. 2011. Tracking leatherback turtles from the world's largest rookery: assessing threats across the South Atlantic. Proceedings of the Royal Society B, 10 pp. <10.1098/rspb.2010.2467>

Worton BJ. 1989. Kernel methods for estimating the utilization distribution in home range studies. Ecology 70: 164-168.

Yasuda T \& N Arai. 2005. Fine-scale tracking of marine turtles using GPS-Argos PTTs. Zoological Science 22: 547-553.

Zavala-Hidalgo J, SL Morey \& JJ O'Brien. 2003. Seasonal circulation on the western shelf of the Gulf of Mexico using a high-resolution numerical model. Journal of Geophysical Research 108 (12): 1-19. 